\title{
The impact of lipoprotein lipase deficiency on health-related quality of life: a detailed, structured, qualitative study
}

\author{
Sasi Neelamekam ${ }^{1,2}$, See Kwok ${ }^{1,2}$, Rachel Malone ${ }^{3}$, Anthony S. Wierzbicki ${ }^{4}$ and Handrean Soran ${ }^{1,2^{*}}$ (D)
}

\begin{abstract}
Background: Lipoprotein lipase deficiency (LPLD) is an autosomal recessive inherited disorder caused by loss-offunction mutations in genes involved in the lipoprotein lipase pathway. It is characterised by chylomicronaemia, severe hypertriglyceridaemia and an increased risk of recurrent pancreatitis that often requires hospitalisation. This research aimed to improve our understanding of the debilitating impact that LPLD has on the daily lives of patients and their families.
\end{abstract}

Methods: The research comprised a 2-h interview with the patient and, where possible, a 1-h interview with a family member; a 1-week pre- and post-interview task (written and/or video diary); and a 30-45-min follow-up telephone interview. Feelings and thoughts at each stage of the disease journey were captured on a 0-10 rating scale, while the impact of disease on overall health status was measured via the EuroQoL 5 domains, 3 levels (EQ-5D-3L) questionnaire (descriptive and visual analogue scale).

Results: Of four patients identified, three (two female, one male) were recruited to participate in the study; the male patient did not complete the pre-interview task or consent to a family member interview. Demographics and medical history differed among patients in terms of age at symptom onset, their journey to LPLD diagnosis, treatments, the number of attacks of pancreatitis and lengths of hospitalisations. Health-related quality of life, assessed by the EQ-5D-3L, was poor during acute attacks of pancreatitis but was minimally impacted by their condition at interview. Patients described feeling apprehensive, frightened, anxious, depressed or frustrated during and after hospitalisations; spouses of the two female patients also reported being worried or afraid. LPLD affected many aspects of daily living, including diet; socialising and building relationships; state of mind (fear of another attack of pancreatitis or lack of disease control); college and working life (through absenteeism and consequent financial implications); and being reliant on family and friends for support.

Conclusions: The interviews of the three patients with LPLD highlighted several concerns and emphasised the need for improved education, support, dietary advice and appropriate disease management. Additional support services would ease the fear and uncertainty surrounding attacks of pancreatitis, and would allow for improved treatment during hospitalisations.

Keywords: Burden of disease, Chylomicronaemia, Health-related quality of life, Hypertriglyceridaemia, Lipoprotein lipase deficiency, Pancreatitis

\footnotetext{
* Correspondence: Hsoran@aol.com

${ }^{1}$ Cardiovascular Trial Unit, Central Manchester University Hospitals NHS

Foundation Trust, Manchester, UK

2Division of Cardiovascular Sciences, University of Manchester, Manchester,

UK

Full list of author information is available at the end of the article
} 


\section{Background}

Familial lipoprotein lipase deficiency (LPLD, sometimes known as chylomicronaemia syndrome) is caused by loss-of-function mutations in genes involved in the lipoprotein lipase pathway and is the result of autosomal recessive inheritance [1]. Clinical features include severe hypertriglyceridaemia, chylomicronaemia and an increased risk of recurrent pancreatitis [2, 3]. The estimated prevalence of LPLD is approximately 1 per million population $[1,3]$, which equates to about 65 people living with LPLD in the UK [4]. The majority of individuals with familial LPLD develop symptoms before 10 years of age, and about $25 \%$ of those affected show symptoms before the age of 1 year [1, 3]. Males and females are affected equally [3].

Lipoprotein lipase is the central enzyme responsible for the breakdown of triglyceride-rich lipoproteins known as chylomicrons [5]. In LPLD, clearance of chylomicrons from plasma is impaired, resulting in the accumulation of chylomicrons and therefore, triglycerides in blood. This accumulation, particularly in capillaries, leads to a build-up of triglycerides in adipose and skeletal tissues $[2,6]$. Indeed, patients with LPLD can have fasting triglyceride levels 10 - to 100-fold greater than normal values $(\leq 1.7 \mathrm{mmol} / \mathrm{L})$ [6]. The 'chylomicron plugs' in capillary beds and corresponding high triglyceride levels lead to the signs and symptoms characteristic of the condition, including abdominal pain, which is usually a result of acute pancreatitis, eruptive xanthomata, hepatosplenomegaly, lipaemia retinalis, peripheral neuropathy and cardiopulmonary symptoms [1, 3, 7].

The risk of acute pancreatitis increases with plasma triglyceride levels above $10 \mathrm{mmol} / \mathrm{L}$ [8-10]. Pancreatitis associated with LPLD is often recurrent and unpredictable, and can be severe and necrotising [11, 12], with attacks that typically occur at a younger age than with non-hereditary causes of hypertriglyceridaemia [13, 14]. Irrespective of the cause, recurrent episodes of acute pancreatitis may culminate in chronic disease. This can increase the risk of exocrine and endocrine insufficiency resulting from the destruction of pancreatic parenchyma, leading to diabetes $[6,13]$. Patients experiencing an attack of pancreatitis often require hospitalisation to control pain and to treat complications, such as multiple organ failure [13]. Severe attacks can be fatal: intensive care unit and in-hospital mortalities of $31 \%$ and $42 \%$, respectively, have been reported in the UK, compared with mortality as low as $1 \%$ for mild pancreatitis $[11,15]$. These attacks may also include a variety of associated medical problems and impaired psychosocial functioning [11, 13, 16, 17].

The mainstay of treatment for LPLD is a very low fat diet, which aims to decrease fasting triglyceride levels to below or close to $10 \mathrm{mmol} / \mathrm{L}$ by restricting the amount of dietary fat to $20 \mathrm{~g} /$ day or less (or $15 \%$ of the total energy intake) [3, 18]. Nevertheless, almost $30 \%$ of patients with LPLD develop pancreatitis despite adhering to such a diet [14]. During acute attacks, temporary cessation of food intake with intravenous fluid hydration can be used to stop the production of post-prandially secreted chylomicrons from the gut. In severe acute pancreatitis, which often requires hospitalisation and sometimes intensive care unit admission [19], excess chylomicrons can be removed physically by plasmapheresis or haemodialysis [18].

The symptoms of LPLD, the need to follow a fatrestricted diet and the impaired psychosocial functioning associated with the disease have an impact on healthrelated quality of life (HRQoL) [7, 20]. Furthermore, a lack of effective therapies for LPLD is likely to add to the burden of disease. This research aims to improve our understanding of the impact that LPLD has on patients and their relatives, and to demonstrate clearly the challenges these people face in daily life. This was achieved through detailed, structured interviews with three patients in the UK and their family members.

\section{Methods \\ Ethical approval}

The study (IRAS Project ID: 173627; REC reference number: 16/YH/022; NIHR CRN reference: META 30909) was approved by the Yorkshire and the Humber - Sheffield Research Ethics Committee. Informed consent to participate was obtained from eligible patients and all participating family members; ethical approval was granted for inclusion of a maximum of four patients. According to the protocol and the conditions laid down by the ethics committee, participants confirmed that the case studies were an accurate representation of their interview and were made aware that the anonymised results may be published in peer-reviewed journals. The ethics committee agreed that no further participant consent would be required for publications originating from the anonymised case studies.

\section{Patients}

Patients were identified (screened) from a review of their medical notes at two tertiary lipid clinic databases to confirm that they met the research inclusion criteria. Potential participants were subsequently contacted by their regular LPLD clinician and were invited to join the study to enable three patient case examples to be investigated. All those identified were approached for consent.

Individuals interested in participating in the study were sent patient information sheets and informed consent forms. Those who required additional information or wished to proceed were asked to contact Synergy Healthcare Research, London, UK (the clinical research organisation commissioned by the study sponsor to conduct the research, including all interviews). Patients were informed that their research documentation would only 
be accessible to Synergy Healthcare Research, and that neither their name nor any other identifiable information would be disclosed outside of this organisation unless explicit consent was obtained. Although patients were not informed of the identity of the study sponsor, they were told that a pharmaceutical company was sponsoring the research.

Patients who were eligible to take part in the study underwent additional screening by Synergy Healthcare Research to ensure that they met the inclusion criteria. Male or female adult patients (aged 18 years or older) with a clinical diagnosis of LPLD confirmed by genetic testing were eligible for study inclusion. Patients also had to have fasting triglyceride levels above $20 \mathrm{mmol} / \mathrm{L}$ at the time of screening and a history of acute pancreatitis or abdominal pain consistent with pancreatitis. Patients with secondary causes of hypertriglyceridaemia (e.g., excess alcohol intake or uncontrolled diabetes) were excluded from the study.

\section{Study design and interviews}

The study was based on interviews with the patient and, where agreed, a family member, in addition to diary entries from the patient. Overall, the research comprised five elements: a 2 -h face-to-face interview with the patient; where possible, a 1-h face-to-face interview with the patient's family member (held on the same day as the patient interview); a 1-week pre- and a postinterview task; and a 30-45-min follow-up telephone interview. For the 1-week pre- and post-interview task, patients were requested to complete a written and/or video diary at the end of each day. Participants were asked to spend at least 2-3 min recording the impact that LPLD had on their life on that particular day. They were advised to consider how they felt, any symptoms that they had, how LPLD and/or their symptoms had affected them and their family/friends, the food they had eaten or avoided, effects on general activities of daily living and the impact of their condition at work. The face-to-face interview was the minimum level of participation a patient was required to consent to in order to take part in the study. All other tasks were optional (including the interview with the family member). Patients and family members were offered $£ 40-£ 80$ per task as compensation for their participation.

During the face-to-face interview, time was spent exploring the patient journey from symptom onset, through diagnosis and previous/current treatments. The effect of symptoms or acute episodes of pancreatitis formed a key discussion point. Feelings and thoughts at each stage of the patient journey and during periods of significant symptoms or episodes of acute pancreatitis were captured using showcards with a 0-10 rating scale and a combination of words, colours or faces. The patient was also asked to complete the EuroQoL 5 domains, 3 levels (EQ-5D-3L) questionnaire [21]. This is a standardised instrument for use as a measure of health outcome and provides a simple descriptive profile and a single index value for health status [21]. The EQ-5D-3L employs a descriptive system, in which the patient is required to indicate his/her health state by ticking one of three boxes against the most appropriate statement (1, no problem; 2 , some problems; 3 , extreme problems) for each of the five dimensions assessed (i.e., mobility, self-care, usual activities [work, study, housework, family and leisure activities], pain/discomfort and anxiety/depression). This questionnaire also uses a visual analogue scale (VAS; score of $100=$ best state imaginable; score of $0=$ worst state imaginable) [21] to quantify the impact of LPLD on overall health status. In each case, the patient was asked to consider two different scenarios: (i) how they remembered feeling during their most severe acute attack of pancreatitis; and (ii) how they felt at the time of the interview.

\section{Analysis}

Data were analysed descriptively. To obtain overall mean scores in the EQ-5D-3L and the VAS for all three patients, the mean of individual ratings for each patient was calculated for each time point (during the most severe attack compared with at the time of the interview).

\section{Results}

\section{Patient demographics and medical history}

Of four patients identified and screened, three were recruited (two from Manchester and one from London, UK) to participate in the study (patients 1, 2 and 3). One patient did not meet full entry requirements when completing the screening questionnaire because he did not answer any question directly and instead referred all questions to his doctor. Two of the three recruited patients completed the pre-interview diary, and all three completed the face-to-face interview, post-interview diary and follow-up telephone interview. For patients 1 and 3, a family member was also interviewed; however, patient 2, who did not complete the pre-interview diary, did not consent to having a family member interviewed.

Patient demographics and medical history are presented in Table 1. The age at symptom onset and LPLD diagnosis varied among the three patients. The journey to diagnosis for patient 1 , the oldest participant, was long and complex. She first developed symptoms at the age of 13 years and was later (at 18 years old) diagnosed with hyperlipidaemia type I/V and acute pancreatitis; however, she did not receive a confirmed diagnosis of LPLD until more than 30 years later. Patient 2 was the youngest of the three patients included in the study. $\mathrm{He}$ first developed symptoms when at primary school; he 
Table 1 Patient demographics and medical history, based on patients' recollections of events

\begin{tabular}{|c|c|c|c|}
\hline & Patient 1 & Patient 2 & Patient 3 \\
\hline Sex & Female & Male & Female \\
\hline Age (years) & 64 & 28 & 42 \\
\hline Age at LPLD symptom onset (years) & 13 & Primary school age & Early 30 s \\
\hline Age at diagnosis of LPLD (years) & $\begin{array}{l}\text { Early } 50 \text { s (diagnosis of } \\
\text { hyperlipidaemia type IN and } \\
\text { acute/chronic pancreatitis at } \\
18 \text { years old) }\end{array}$ & Young age & Early $40 \mathrm{~s}$ \\
\hline $\begin{array}{l}\text { Number of acute attacks of } \\
\text { pancreatitis requiring hospitalisation }\end{array}$ & $>15^{\mathrm{a}}$ & $\begin{array}{l}102^{\mathrm{b}} \text { (requiring hospitalisation } \\
\text { over } 5 \text { years) }\end{array}$ & $\begin{array}{l}5 \text { (2 admissions to ITU; } \\
2 \text { to HDU; } 1 \text { admission } \\
\text { to a ward) }\end{array}$ \\
\hline $\begin{array}{l}\text { Medications }{ }^{c} \text { taken at home for } \\
\text { LPLD symptoms }\end{array}$ & Antox ${ }^{\oplus}$, black garlic & $\begin{array}{l}\text { Tramadol, cyclizine, Deep } \\
\text { Heat }{ }^{\oplus} \text { patches }\end{array}$ & $\begin{array}{l}\text { Statins, fenofibrate, } \\
\text { omega-3 capsules }\end{array}$ \\
\hline $\begin{array}{l}\text { In-hospital medications/management } \\
\text { during attacks of pancreatitis }\end{array}$ & $\begin{array}{l}\text { IV fluids and morphine, slow } \\
\text { re-introduction of food }\end{array}$ & $\begin{array}{l}\text { IV fluids, morphine and } \\
\text { cyclizine followed by IV } \\
\text { paracetamol, nil by mouth } \\
\text { for } 1-2 \text { days }\end{array}$ & $\begin{array}{l}\text { Analgesic medications, } \\
\text { total parenteral nutrition }\end{array}$ \\
\hline Comorbidities & PVD, hypertension, IBS & None & Type 2 diabetes \\
\hline Family members with LPLD & $\begin{array}{l}\text { Sister had a child (son) who died } \\
\text { from high levels of lipids in the } \\
\text { liver (formal diagnosis unknown) }\end{array}$ & Yes (sister) & Yes (sister) \\
\hline
\end{tabular}

HDU high-dependency unit, IBS irritable bowel syndrome, ITU intensive therapy unit, IV intravenous, LPLD lipoprotein lipase deficiency, PVD peripheral vascular disease

${ }^{a}$ This number may be underestimated given that patient 1 experienced one attack per month in her late teens and early $20 \mathrm{~s}$

${ }^{\mathrm{b}}$ This number was estimated by the patient

'Pharmacological and alternative

was diagnosed with LPLD as a young child, following the diagnosis of an older sister. Patient 3 had a succession of recurrent severe acute attacks of pancreatitis in her mid-to-late 30s, which occurred over a relatively short period of time; a diagnosis of LPLD was given 3 years later.

All three patients follow a fat-restricted diet. With the exception of dietary intervention, medications taken at home differed among the three patients (Table 1). Patient 2 was also receiving investigative therapy for his condition.

Patient 3 has two children, who were born between her diagnosis of diabetes and the development of her first symptoms of LPLD. Patient 1 was sterilised in her late teens following the diagnosis of hyperlipidaemia type I/V after being told she should not have children because of her illness. Patient 2 has no children.

All three patients had similar symptoms, including acute pancreatitis, chronic pancreatitis (patients 1 and 2 only), abdominal pain, vomiting and nausea, back pain and plasma lactescence. Patient 1 also had hepatosplenomegaly and new-onset cardiovascular disease, while patient 3 additionally had steatorrhoea. Reported comorbidities were generally cardiometabolic in nature, except for concomitant irritable bowel syndrome, reported by patient 1 . Patient 3 also had type 2 diabetes, which was diagnosed in her early 30 s.
The number of acute attacks of pancreatitis requiring hospitalisation in these patients also differed (Table 1). Patient 1 estimated that she had had over 15 attacks requiring hospitalisation. However, this number may be underestimated given that she reported experiencing one attack per month in her late teens and early 20 s. Patient 2 had the impression that he had had a considerable number of acute attacks requiring hospitalisation (estimated at 102 admissions in total). He reported that attacks increased in severity as he became older. Patient 3 reported that she had had five attacks requiring hospitalisation (one mild, four severe); she was the only patient admitted to the intensive therapy unit (ITU) and high-dependency unit during hospitalisations. The longest periods of hospitalisation were reported to be 15 weeks (3 weeks in a local hospital followed by 12 weeks in a metabolic unit), 5 days and 2 weeks for patients 1,2 and 3 , respectively.

\section{Impact of LPLD on HRQoL}

All three patients remembered that their HRQoL was poor during the worst acute attack of pancreatitis, as assessed by the EQ-5D-3L. However, they confirmed that their HRQoL was minimally impacted by their condition at the time of the interviews. The average health status of all three patients across four of the five domains (mobility, self-care, usual activities and pain/ discomfort) of the EQ-5D-3L descriptive system was level 
3 during acute attacks and level 1 at the time of the interview. The average health status for anxiety/depression was level 1.7 during acute attacks and level 1 at the time of the interview. When the average health status of all three patients was assessed using the VAS, it was 14.7 during acute attacks and 90.0 at the time of the interview (see Additional file 1 for individual scores).

Figure 1 depicts a timeline of feelings at key points in the disease journey. Patient 1 did not believe that her HRQoL had been affected by LPLD; she has a very positive attitude and reported that she did not allow her illness to control her. She confirmed that she leads a healthy and active lifestyle alongside her spouse. During her teens/early adulthood, when she experienced many acute attacks of pancreatitis, she reported remaining unperturbed, despite believing that she did not have long to live. This patient, however, did describe feeling apprehensive during the 15-week hospitalisation following an attack of pancreatitis in her 30s (Fig. 1a). Her husband is also very pragmatic, but described worrying about a potential attack whenever his wife has a stomach ache or back pain.

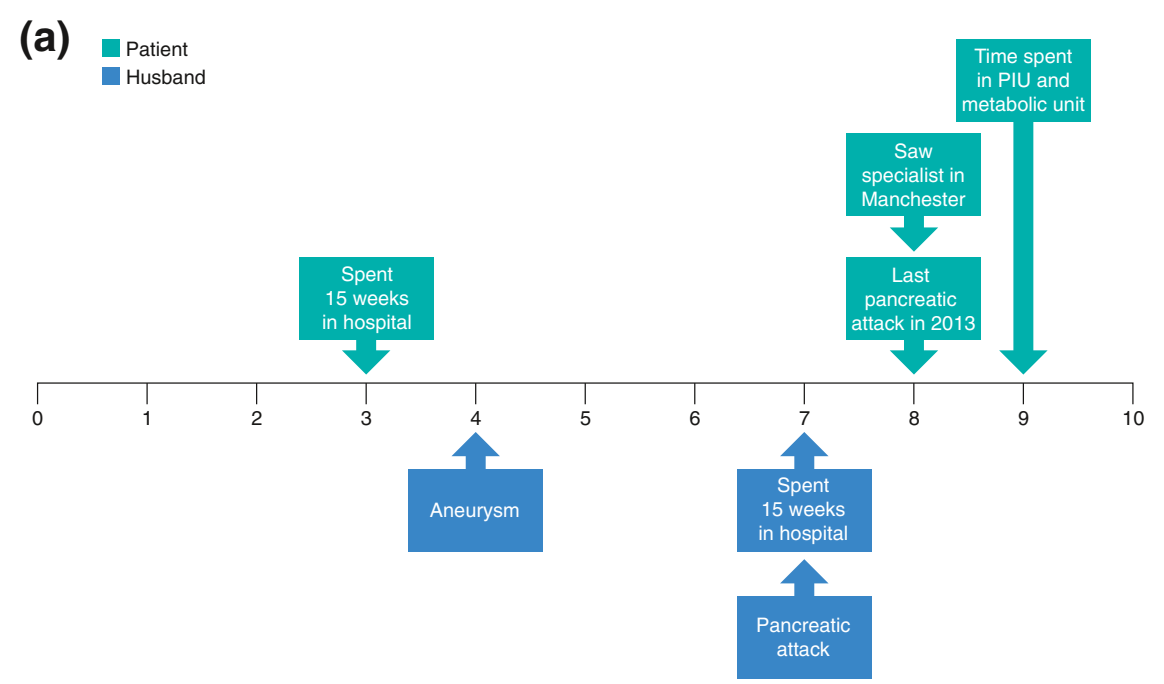

(b)
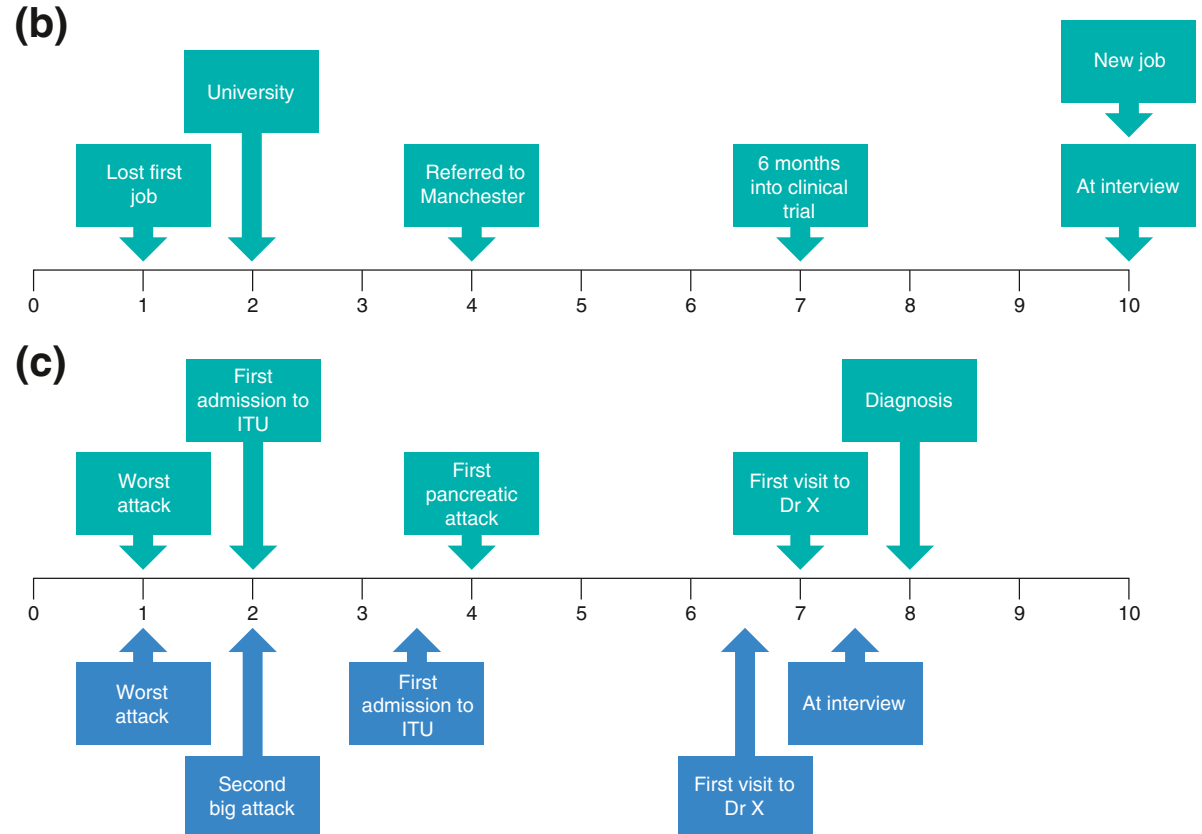

Fig. 1 Timeline of feelings: a Patient 1 and her husband; $\mathbf{b}$ patient 2; $\mathbf{c}$ patient 3 and her husband. Feelings at key points in the patient's and family member's disease journey for the three patients were rated using a score of 0 (extremely negative) to 10 (extremely positive). ITU intensive therapy unit; PIU programmed investigation unit 
By contrast, patients 2 and 3 reported periods of depression and anxiety (Fig. $1 \mathrm{~b}$ and $\mathrm{c}$ ). Patient 2 described a 6-month period of depression following two acute attacks of pancreatitis that occurred during his first job after finishing university:

"That was the worst where I was just at home with nothing literally... I felt it was my prime time and I was losing it all to this [the illness] and I had no answers from anybody."

This patient also reported feeling stressed and frustrated during hospitalisations, having to explain to medical staff that his condition was not alcohol related:

"That was the worst thing, when you felt like death ... I used to say straightaway, it's not alcohol related, I had this from birth. But it never really sank in."

Patient 3 provided detailed accounts of her emotions and described being "frightened, worried and alone" during her first ITU stay, following a severe attack. In between hospitalisations, patient 3 reported being constantly worried that she would experience another attack, and feeling miserable and frustrated that nobody could do anything to prevent it.

"I worried every day about it [having another attack] and it's the question of when's this going to happen again, not if."

After her worst attack (which led to ITU admission for 2 weeks), patient 3 was very frightened: “... most terrified I've ever been in my life". It was after this period, following discharge, that she reported becoming increasingly anxious and depressed, not only owing to the fear of recurrent pancreatitis episodes and hospitalisations but also because of a lack of control over her illness. Her husband also described feeling "frightened, concerned and nervous" following his wife's worst attack.

\section{Burden of disease}

In addition to the burden of illness experienced by the three patients and their families, LPLD was described as having a substantial psychological impact, driven by fear or the threat of another attack and by the impact that the disease had on daily living. A key area for all three patients was adhering to a strict diet, which they found to be challenging. The dietary interventions placed upon patients with LPLD also result in limitations with respect to the use of convenience foods and eating out owing to the uncertainties of dietary composition of foods. Patient 1 described spending a considerable amount of her daily routine preparing and cooking meals. One of her key concerns surrounded the fear of being unable to control her diet later in life when she might not be able to cook for herself. Patient 2 described having to forego his mother's cooking, which had led to tensions in the family and having to resort to a "boring" and easily digestible diet, such as chicken, tuna and pasta. Importantly, patients stated that they had generally received little useful dietary support from their healthcare professionals.

LPLD also influenced the university and working life of these patients. Patient 2 reported that his illness has affected his education, career, and social and personal life. The increased frequency of pancreatitis and hospitalisations during his time at university meant that he was ill for $\sim 50 \%$ of this time; consequently, this probably affected his degree grade. Patients 1 and 2 did not disclose their illness to employers until attacks of pancreatitis resulted in hospitalisations. For all patients, employers have generally been supportive and understanding. However, having LPLD resulted in recurrent work absenteeism - sometimes for extended periods (up to 33 weeks for patient 1), reduced/part-time working hours and, subsequently, financial concerns (particularly for patients 2 and 3 ).

Patients described having to rely on their families or friends for support during attacks and hospitalisations. However, a lack of understanding of the seriousness and cause of the disease from family members sometimes led to raised anxiety or tension during attacks. For example, patient 3 reported that she had the impression that her parents thought the attacks were self-inflicted because she was not adhering to her diet, while patient 2 described keeping his family "out-of-the-loop" to avoid stressful situations. Patient 1 described how, during attacks in her teenage years, her father did not tell her mother of the attacks to avoid worrying her; consequently, the mother was not informed of hospitalisations and patient 1 did not receive any visitors during this stage.

Other issues reported included the need to live in certain locations, as in the case of patient 1 who was advised that diesel fumes could exacerbate her condition; as a result, she lives in a remote area to avoid fumes. Patient 3 faces the challenge of having to decide whether or not to have her children tested for LPLD and the impact that a positive diagnosis would have for them.

\section{Unmet needs}

Table 2 lists key areas of unmet need identified by these three patients. One of the main concerns was the lack of knowledge and understanding of LPLD and pancreatitis by healthcare professionals. Patients and family members reported having to conduct much of their own research into LPLD because they felt that the information they were receiving from their general physicians and other healthcare professionals on the disease was inadequate. In some cases, patients/spouses had to inform medical staff 
Table 2 Unmet needs highlighted by patients with lipoprotein lipase deficiency

\begin{tabular}{|c|c|c|}
\hline Patient 1 & Patient 2 & Patient 3 \\
\hline $\begin{array}{l}\text { - Medical education and support from HCPS } \\
\text { - Ensure that medical records are passed } \\
\text { to relevant onward care providers }\end{array}$ & $\begin{array}{l}\text { - Medical education and support from HCPs } \\
\text { - Early access to disease expert/specialist } \\
\text { - Early access to effective and well-tolerated } \\
\text { treatments } \\
\text { - Advice and emotional support for patients } \\
\text { and family members }\end{array}$ & $\begin{array}{l}\text { - Dietary advice } \\
\text { - Better understanding of pancreatitis } \\
\text { - Prompt diagnosis } \\
\text { - HCP support during long gaps between } \\
\text { outpatient appointments } \\
\text { - Emotional support } \\
\text { - Information to aid patient and family disease } \\
\text { knowledge/understanding }\end{array}$ \\
\hline
\end{tabular}

HCP healthcare professional

upon hospital admission that they suspected an acute attack of pancreatitis to facilitate appropriate care. In relation to this lack of understanding, patients described how appropriate treatment was often delayed during hospitalisations. As previously mentioned, patients were occasionally perceived to have alcoholism when they presented at hospital with symptoms, causing further distress and delays in treatment. In addition, healthcare professionals often gave insufficient, inconsistent and/or inappropriate dietary advice, making it difficult for patients to adhere to the required diet and thereby control their disease. Lack of patient and family support, and understanding from the wider healthcare community was also highlighted by patients, who reported a high unmet need for emotional support and counselling.

\section{Discussion}

The case reports presented here give an insight into daily life for patients with LPLD and their family members. The individuals described in this study are currently controlling their condition through a fat-restricted diet, and, in the case of patient 2 , with additional investigative therapy. However, the daily challenges they face, as well as the acute attacks of pancreatitis and associated hospitalisations, are an indication of the experiences of many patients with LPLD. It should be remembered that despite adhering to a fat-restricted diet, almost $30 \%$ of individuals with LPLD develop pancreatitis [14]. Furthermore, the patients described fear and anxiety associated with attacks of pancreatitis. There is clearly an unmet need for healthcare services to provide support and education for people with this condition.

While the presenting symptoms of LPLD appeared to be relatively consistent among the patients in this study (acute/chronic pancreatitis, abdominal pain, vomiting and nausea, back pain and plasma lactescence), there was undoubtedly much variation in other aspects of the disease profile. The age at symptom onset, number and severity of attacks and comorbidities, treatments and general patient journey to diagnosis were all different, suggesting that there is no 'typical' patient with LPLD. As might be expected given such differences, each patient dealt with their disease and attacks of pancreatitis differently, and described a range of emotions, from feeling very positive and pragmatic to experiencing fear, depression and anxiety.

Patient 3's two children were born between her diagnosis of diabetes and the development of her first LPLD symptoms. There are published reports of hypertriglyceridaemia and attacks of pancreatitis occurring during pregnancy, after which a diagnosis of LPLD has been established [22-24]. This suggests that pregnancy may aggravate the disease in some women, inducing symptoms that may not have occurred previously or that went unnoticed. Healthcare professionals should be aware of the impact of pregnancy on the development of the disease, and investigate the possibility of a diagnosis if symptoms suggestive of LPLD occur in pregnant women.

LPLD has a significant impact on patients' HRQoL, as demonstrated by results obtained from the EQ-5D-3L tool when assessing four of the five domains (mobility, self-care, usual activities and pain/discomfort). During attacks of pancreatitis, patients gave their HRQoL a low score; in contrast, HRQoL was minimally impacted by their condition at the time of the interviews. The frequency and severity of attacks of pancreatitis resulting in hospitalisations, sometimes for very long periods, affected overall HRQoL. For example, patient 2, who reported experiencing 102 attacks of pancreatitis that required hospitalisation over a 5-year period, scored his health on the EQ-5D-3L VAS during that period as 4/100 (where 0 is the worst state imaginable). The impact of hospitalisations on HRQoL, combined with the uncertainty surrounding the frequency and severity of attacks, is likely to create substantial worry for patients. In particular, this may be the case for patients with young families, who may not have relatives nearby to help and/or who may be concerned about the emotional impact of the hospitalisations on their children. Interestingly, the average health status for anxiety/depression was scored relatively high during acute attacks as well as at the time of the interview (level 1.7 versus level 1 , respectively), even though patients 2 and 3 reported that their condition had resulted in periods of depression and anxiety. Despite the worry and stress reported by patients, they generally try to remain positive throughout episodes of hospitalisation. The low VAS score 
reported during attacks of pancreatitis may be due in part to a reported lack of awareness and limited support from healthcare professionals, resulting in stress and uncertainty in the patients and their family members. LPLD also had a substantial effect on patients' careers; irregular working hours necessitated by the condition are likely to result in financial concerns, as described by the patients in this study.

There is a requirement for improved general education on LPLD among healthcare professionals and a need for increased awareness of symptoms among hospital staff, particularly in those initially encountered by patients during attacks (e.g., accident and emergency department staff) to prevent delays in treatment. To this end, it may be useful to issue diagnosed patients with an identifying medical card or bracelet [25], which would explain their diagnosis and describe emergency treatment steps to healthcare professionals to prevent delays in treatment when they are hospitalised.

There is an unmet need for appropriate and consistent dietary advice, as well as education and sources of information on LPLD for patients and family members. Education of healthcare professionals should be improved to ensure that appropriate diets are followed during hospitalisations and to allow patients to better understand and control their condition through diet at home. There is also an unmet need for appropriate counselling, emotional support services and support groups. Patients and their family members consistently described a high level of fear surrounding attacks of pancreatitis. Provision of such services and materials offering sources of information would help to ease family tensions and anxiety, and enable patients to understand their disease better.

This assessment of HRQoL and the burden of disease in LPLD provides insight into the key unmet needs that patients face. The results reported here reflect those of a recent publication based on a comprehensive online research survey of 60 patients with LPLD [20]. Similar to the current work, Davidson et al. reported that most patients experience a lengthy journey to diagnosis, and substantially reduced HRQoL as a result of their illness [20]. LPLD was also reported to influence patients' career choices and employment status. Furthermore, patients described high levels of anxiety, fear and worry surrounding attacks of pancreatitis. The results reported by Davidson et al. support and strengthen those uncovered in this study, emphasizing the burden of disease and unmet need that patients with LPLD face.

A limitation of this study is the low number of participants. This is an unavoidable limitation resulting from the rarity of the disease and the low numbers of patients with LPLD in the UK, and may limit the interpretations presented. Future research should focus on examining the impact of LPLD on HRQoL in patients from other countries to determine whether the issues raised here are applicable on a broader scale. Some of the key unmet needs identified in this study may not apply in other regions; findings from other countries may therefore provide insights into how to address these issues.

\section{Conclusions}

The interviews with these three patients with LPLD highlighted several concerns, emphasising the need for education, support and appropriate management. Additional support services, such as those described above, would ease the fear and uncertainty surrounding attacks of pancreatitis, and would facilitate improved treatment during hospitalisations.

\section{Additional file}

Additional file 1: $\mathrm{HRQOL}$ during the worst attack of pancreatitis and at the interview, assessed by EQ-5D-3L VAS. Score of $0=$ worst state imaginable; score of $100=$ best state imaginable. EQ-5D-3L EuroQoL 5 domains, 3 levels; HRQoL health-related quality of life; VAS visual analogue scale. (PDF $838 \mathrm{~kb}$ )

\section{Abbreviations}

EQ-5D-3L: EuroQoL 5 domains, 3 levels; HRQoL: Health-related quality of life; ITU: Intensive therapy unit; LPLD: Lipoprotein lipase deficiency; VAS: Visual analogue scale

\section{Acknowledgements}

The authors thank the patients and their families for their participation in this study. All interviews were carried out by Synergy Healthcare Research, funded by Chiesi Limited. Medical writing and editorial support was provided by Noëlle L O'Regan PhD and Sharon Smalley, of PharmaGenesis London, London, UK, and was funded by Chiesi Limited.

\section{Funding}

This study was sponsored by Chiesi Limited. The funding body was involved in the design of the study and analysis and interpretation of the data. One author (RM) is an employee of Chiesi Limited; other employees of the funding body reviewed the manuscript for medical accuracy.

\section{Availability of data and materials}

The data generated during this study are contained in this published article and its supplementary information file.

\section{Authors' contributions}

SN, SK, ASW and HS made substantial contributions to the acquisition of data. SK, ASW, RM and HS conceived the hypothesis and designed the study. All authors made substantial contributions to the analysis and interpretation of data, were involved in drafting and critically revising the manuscript, gave final approval of the version to be published and agree to be accountable for all aspects of the work.

\section{Ethics approval and consent to participate}

The study (IRAS Project ID: 173627; REC reference number: 16/YH/022; NIHR CRN reference: META 30909) was approved by the Yorkshire and the Humber - Sheffield Research Ethics Committee. Informed consent to participate was obtained from eligible patients and all participating family members.

\section{Consent for publication}

Participants confirmed that the case studies were an accurate representation of their interview and were made aware that the anonymised results may be published in peer-reviewed journals. The ethics committee agreed that no 
further participant consent would be required for publications originating from the anonymised case studies.

\section{Competing interests}

SN, SK and ASW have no competing interests. RM is an employee of Chiesi Limited. HS has received research grants from Alexion, Amgen, MSD and Pfizer, and speaker honoraria, personal fees for consultancy and educational grants from Aegerion, AKCEA, Alexion, AstraZeneca, Amgen, Janssen Cilag Limited, Lilly, MSD, Pfizer and Sanofi.

\section{Publisher's Note}

Springer Nature remains neutral with regard to jurisdictional claims in published maps and institutional affiliations.

\section{Author details}

${ }^{1}$ Cardiovascular Trial Unit, Central Manchester University Hospitals NHS Foundation Trust, Manchester, UK. ²Division of Cardiovascular Sciences, University of Manchester, Manchester, UK. ${ }^{3}$ Chiesi Limited, Manchester, UK. ${ }^{4}$ Guy's and St Thomas' Hospitals, London, UK.

\section{Received: 27 June 2017 Accepted: 5 September 2017}

\section{Published online: 19 September 2017}

\section{References}

1. National Library of Medicine (US). Familial lipoprotein lipase deficiency. https://ghr.nlm.nih.gov/condition/familial-lipoprotein-lipasedeficiency\#statistics. Accessed 14 Mar 2017.

2. Gaudet D, Methot J, Dery S, Brisson D, Essiembre C, Tremblay G, Tremblay K, de Wal J, Twisk J, van den Bulk N, et al. Efficacy and long-term safety of alipogene tiparvovec (AAV1-LPLS447X) gene therapy for lipoprotein lipase deficiency: an open-label trial. Gene Ther. 2013;20:361-9.

3. Brunzell JD. Familial lipoprotein lipase deficiency. In: Pagon RA, Adam MP, Ardinger HH, et al., editors. GeneReviews ${ }^{\circledR}$ [Internet]; 1999. https://www.ncbi. nlm.nih.gov/books/NBK1308/. Accessed 14 Mar 2017.

4. Office of National Statistics. Population estimates for UK, England and Wales, Scotland and Northern Ireland: mid-2015. Published June 2016. https:// www.ons.gov.uk/peoplepopulationandcommunity/populationandmigration/ populationestimates/bulletins/annualmidyearpopulationestimates/mid2015. Accessed 14 Mar 2017

5. Goldberg IJ. Lipoprotein lipase and lipolysis: central roles in lipoprotein metabolism and atherogenesis. J Lipid Res. 1996;37:693-707.

6. Gaudet D, Methot J, Kastelein J. Gene therapy for lipoprotein lipase deficiency. Curr Opin Lipidol. 2012;23:310-20.

7. Johnson C, Stroes ES, Soran H, Wierzbicki A, Moulin P, Bruckert E, Steinhagen-Thiessen E, Gaudet D, lotti G, Rastelletti I, et al. Issues affecting quality of life and disease burden in lipoprotein lipase deficiency (LPLD) first step towards a pro measure in LPLD. Value Health. 2015;18:A707.

8. Miller M, Stone NJ, Ballantyne C, Bittner V, Criqui MH, Ginsberg HN, Goldberg AC, Howard WJ, Jacobson MS, Kris-Etherton PM, et al. Triglycerides and cardiovascular disease: a scientific statement from the American Heart Association. Circulation. 2011;123:2292-333.

9. Gaudet D, de Wal J, Tremblay K, Dery S, van Deventer S, Freidig A, Brisson D, Methot J. Review of the clinical development of alipogene tiparvovec gene therapy for lipoprotein lipase deficiency. Atheroscler Suppl. 2010;11:55-60.

10. Scherer J, Singh VP, Pitchumoni CS, Yadav D. Issues in hypertriglyceridemic pancreatitis: an update. J Clin Gastroenterol. 2014;48:195-203.

11. Pavlidis P, Crichton S, Lemmich Smith J, Morrison D, Atkinson S, Wyncoll D, Ostermann M. Improved outcome of severe acute pancreatitis in the intensive care unit. Crit Care Res Pract. 2013;2013:897107.

12. Sandhu S, Al-Sarraf A, Taraboanta C, Frohlich J, Francis GA. Incidence of pancreatitis, secondary causes, and treatment of patients referred to a specialty lipid clinic with severe hypertriglyceridemia: a retrospective cohort study. Lipids Health Dis. 2011;10:157.

13. Bruno MJ. Gene therapy coming of age - prevention of acute pancreatitis in lipoprotein lipase deficiency through alipogene tiparvovec. Eur Gastroenterol Hepatol Rev. 2010;6:48-53.

14. Simon P, Weiss FU, Zimmer KP, Koch HG, Lerch MM. Acute and chronic pancreatitis in patients with inborn errors of metabolism. Pancreatology. 2001;1:448-56.

15. Harrison DA, D'Amico G, Singer M. Case mix, outcome, and activity for admissions to UK critical care units with severe acute pancreatitis: a secondary analysis of the ICNARC Case Mix Programme Database. Crit Care. 2007;11(Suppl 1):S1.

16. Johnson CD, Besselink MG, Carter R. Acute pancreatitis. BMJ. 2014;349:g4859.

17. Balliet WE, Edwards-Hampton S, Borckardt JJ, Morgan K, Adams D, Owczarski S, Madan A, Galloway SK, Serber ER, Malcolm R. Depressive symptoms, pain, and quality of life among patients with nonalcohol-related chronic pancreatitis. Pain Res Treat. 2012;2012:978646.

18. Wierzbicki AS, Viljoen A. Alipogene tiparvovec: gene therapy for lipoprotein lipase deficiency. Expert Opin Biol Ther. 2013;13:7-10.

19. Yi F, Ge L, Zhao J, Lei Y, Zhou F, Chen Z, Zhu Y, Xia B. Meta-analysis: total parenteral nutrition versus total enteral nutrition in predicted severe acute pancreatitis. Intern Med. 2012;51:523-30.

20. Davidson M, Stevenson M, Hsieh A, Ahmad Z, Crowson C, Witztum JL. The burden of familial chylomicronemia syndrome: interim results from the INFOCUS study. Expert Rev Cardiovasc Ther. 2017;15:415-23.

21. EQ-5D questionnaire and visual analogue scale. https://euroqol.org/. Accessed 14 Mar 2017.

22. Henderson H, Leisegang F, Hassan F, Hayden M, Marais D. A novel Glu421Lys substitution in the lipoprotein lipase gene in pregnancy-induced hypertriglyceridemic pancreatitis. Clin Chim Acta. 1998;269:1-12.

23. Murugasu CG, Armstrong G, Creedon G, Cavanna JS, Galton DJ, Tomkin GH. Acute hypertriglyceridaemic pancreatitis in a pregnant Indian: a new lipoprotein lipase gene mutation. J R Soc Med. 1998;91:205-7.

24. Suga S, Tamasawa N, Kinpara I, Murakami H, Kasai N, Onuma T, Ikeda Y, Takagi A, Suda T. Identification of homozygous lipoprotein lipase gene mutation in a woman with recurrent aggravation of hypertriglyceridaemia induced by pregnancy. J Intern Med. 1998;243:317-21.

25. MedicAlert. http://www.medicalert.org.uk/. Accessed 10 Apr 17.

\section{Submit your next manuscript to BioMed Central and we will help you at every step:}

- We accept pre-submission inquiries

- Our selector tool helps you to find the most relevant journal

- We provide round the clock customer support

- Convenient online submission

- Thorough peer review

- Inclusion in PubMed and all major indexing services

- Maximum visibility for your research

Submit your manuscript at www.biomedcentral.com/submit
) Biomed Central 